\title{
A 44-Week Open-Label Study Evaluating Safety and Efficacy of Topical Glycopyrronium Tosylate in Patients with Primary Axillary Hyperhidrosis
}

\author{
Dee Anna Glaser ${ }^{1}$. Adelaide A. Hebert ${ }^{2}$. Alexander Nast ${ }^{3,4}$ - William P. Werschler ${ }^{5}$. Lawrence Green ${ }^{6}$. \\ Richard D. Mamelok ${ }^{7} \cdot$ John Quiring $^{8} \cdot$ Janice Drew $^{9} \cdot$ David M. Pariser $^{10}$
}

Published online: 20 May 2019

(c) The Author(s) 2019

\begin{abstract}
Background Glycopyrronium tosylate is a topical anticholinergic approved in the USA for primary axillary hyperhidrosis in patients aged $\geq 9$ years ( $\mathrm{Qbrexza}{ }^{\mathrm{TM}}$ [glycopyrronium] cloth, $2.4 \%$ ).

Objective This 44-week open-label extension study assessed glycopyrronium tosylate safety and descriptive efficacy in patients completing one of two, phase III, double-blind, vehicle-controlled, 4-week trials (NCT02530281; NCT02530294). Methods Patients aged $\geq 9$ years with primary axillary hyperhidrosis were randomized 2:1 (glycopyrronium tosylate: vehicle, once daily) in the double-blind trials. Completers could receive open-label glycopyrronium tosylate for up to an additional 44 weeks. Treatment-emergent adverse events and local skin reactions were assessed. Descriptive efficacy assessments were gravimetrically measured sweat production, Hyperhidrosis Disease Severity Scale responder rate ( $\geq 2$ grade improvement), and Dermatology Life Quality Index/children's Dermatology Life Quality Index.

Results Of 651 patients completing the double-blind trials, $564(86.6 \%)$ entered the open-label extension; 550 were analyzed. Most patients experiencing treatment-emergent adverse events had mild or moderate events $(>90 \%)$. Discontinuation because of treatment-emergent adverse events remained low and relatively stable, with a cumulative rate of $8.0 \%(44 / 550)$ over 44 weeks. Common treatment-emergent adverse events (>5\%) were dry mouth (16.9\%), vision blurred (6.7\%), application-site pain (6.4\%), nasopharyngitis (5.8\%), and mydriasis (5.3\%). Most patients (67.5\%) had no local skin reactions; those occurring were predominantly mild/moderate. Glycopyrronium tosylate efficacy was maintained throughout the trial; at week 44, the Hyperhidrosis Disease Severity Scale responder rate was 63.2\%, and improvements from baseline (double blind) in sweat production were $-71.3 \%$ and $8.7 \pm 6.2 / 6.2 \pm 4.9$ for Dermatology Life Quality Index/children's Dermatology Life Quality Index.

Conclusions Daily long-term application of glycopyrronium tosylate for up to 48 weeks (double blind plus open label) was generally well tolerated and efficacy was maintained. No new safety signals emerged.
\end{abstract}

Trial Registry Clinicaltrials.gov NCT02553798.

\section{Introduction}

Hyperhidrosis, characterized by excess sweat production beyond that which is necessary to maintain thermal homeostasis, affects an estimated $4.8 \%$ of the US population, or approximately 15.3 million people [1,2]. The substantial

Electronic supplementary material The online version of this article (https://doi.org/10.1007/s40257-019-00446-6) contains supplementary material, which is available to authorized users.

Dee Anna Glaser

glasermd@slu.edu

Extended author information available on the last page of the article negative impact of hyperhidrosis on quality of life has been equated as comparable to, or greater than, psoriasis or eczema [3].

Despite the well-established burden on quality of life, hyperhidrosis remains under-recognized as a treatable medical condition [4], which may be attributable, in part, to a number of factors, including lack of disease awareness, patients' inability to recognize symptoms as a medical condition, and/or dissatisfaction with or lack of access to available therapies $[1,4,5]$.

Antiperspirants (over the counter and prescription), anticholinergic drugs (including oral glycopyrrolate and oxybutynin), botulinum toxins, iontophoresis (application 


\section{Key Points}

Glycopyrronium tosylate is the only topical anticholinergic approved in the USA for the treatment of primary axillary hyperhidrosis in adult and pediatric patients $\geq 9$ years of age (Qbrexza ${ }^{\mathrm{TM}}$ [glycopyrronium] cloth, $2.4 \%$, for topical use).

In this long-term, open-label extension trial, safety results were consistent with the safety profile observed in the 4-week, double-blind, controlled, phase III trials of glycopyrronium tosylate, with no new or unexpected findings.

Most treatment-emergent adverse events were related to anticholinergic activity and mild or moderate in severity, and there was a low rate of discontinuation because of treatment-emergent adverse events.

Efficacy assessments throughout the 44-week open-label extension indicate that patients maintained reductions in sweat production and disease severity (Hyperhidrosis Disease Severity Scale), as well as quality-of-life improvements.

of mild electrical current through water), microwave thermolysis, and ablation (e.g., curettage or liposuction) of the sweat glands are used to treat hyperhidrosis [2, 4]. Despite these therapeutic options, patients have historically remained dissatisfied with treatment [4-6], underscoring the need for new treatment approaches. In June 2018, the topical anticholinergic glycopyrronium tosylate (GT) was approved by the US Food and Drug Administration for the treatment of primary axillary hyperhidrosis in adult and pediatric patients 9 years of age and older (Qbrexza ${ }^{\mathrm{TM}}$ [glycopyrronium] cloth, $2.4 \%$, for topical use) [7]. Subsequent to GT approval, the International Hyperhidrosis Society published an updated treatment algorithm (developed by leading dermatologists with hyperhidrosis expertise) for primary axillary hyperhidrosis in which GT and topical antiperspirants are designated as first-line therapies prior to microwave thermolysis or botulinum toxin A injections or oral anticholinergics [8]. Glycopyrronium tosylate is formulated as a topical solution and applied once daily to the axillae using a premoistened towelette. Results from the two randomized, 4-week, double-blind, vehicle (VEH)-controlled, phase III studies of GT for primary axillary hyperhidrosis (ATMOS-1 [NCT02530281] and ATMOS-2 [NCT02530294]) have previously been reported [9-11]. In these trials, GT was generally well tolerated and significantly reduced sweating severity and sweat production compared with VEH along with corresponding improvements in quality of life through 4 weeks of treatment.

Patients who completed week 4 of the phase III, doubleblind, controlled GT trials were eligible to enter the 44-week, open-label extension study (ARIDO, NCT02553798). The primary objective of the open-label extension was to assess the long-term safety of topical GT in a minimum of 100 patients with primary axillary hyperhidrosis who had received GT for at least 12 months (4-week double-blind treatment plus 44-week open-label treatment). Long-term safety and efficacy results from this open-label study are presented here.

\section{Materials and Methods}

\subsection{Study Design}

In the double-blind trials, patients with primary axillary hyperhidrosis were randomized $2: 1$ to GT (3.75\% topical solution) or VEH applied once daily to each axilla for 28 days [9]. Patients who completed the double-blind trials with $\geq 80 \%$ treatment compliance were eligible to continue into the open-label extension and receive GT for up to 44 weeks or until early termination by the study sponsor (Fig. 1 of the Electronic Supplementary Material [ESM]). As allowed by the open-label extension protocol and in accordance with regulatory follow-up requirements, the study sponsor terminated the study early when the primary study objective was achieved, namely that at least 100 patients received GT treatment and provided safety data for at least 12 months (4-week double-blind treatment plus 44-week open-label treatment). Patients were assessed in clinics at day 1 (week 4 visit of the double-blind trials) and weeks 2 , $4,8,12,16,20,28,36$, and 44 (end of treatment/early termination $[\mathrm{ET}])$. Patients were contacted via telephone for a safety follow-up at week 45 (study exit). During the phase III clinical trials, product strength was expressed in terms of glycopyrronium tosylate monohydrate content (3.75\%), while the marketed product (Qbrexza ${ }^{\mathrm{TM}}$ [glycopyrronium] cloth, $2.4 \%$, for topical use) is expressed in terms of glycopyrronium content; the products are equivalent [9].

\subsection{Study Patients}

Detailed inclusion and exclusion criteria for the doubleblind trials were fully reported in the primary publication [9]. Briefly, patients were male or non-pregnant female individuals $\geq 9$ years of age, had primary axillary hyperhidrosis for $\geq 6$ months, gravimetrically measured sweat production $\geq 50 \mathrm{mg} / 5 \mathrm{~min}$ in each axilla, an Axillary Sweating Daily Diary/Axillary Sweating Daily Diary-Children severity item (Item 2) score $\geq 4$ (11-point scale) [12], and 
an Hyperhidrosis Disease Severity Scale (HDSS) grade $\geq 3$ (4-point scale). Patients were excluded for known history of a condition that could cause secondary hyperhidrosis, prior surgical procedure or treatment with a medical device for axillary hyperhidrosis, and prior treatment with iontophoresis or botulinum toxin within 4 weeks and 1 year of baseline, respectively. Male or non-pregnant, non-lactating female patients who completed week 4 of the double-blind trials with $\geq 80 \%$ treatment compliance were eligible to enter the open-label extension if they had signed informed consent and were willing to comply with the protocol. Patients were excluded from the open-label extension for a clinically significant abnormality on physical examination, vital sign, or electrocardiogram at the week 4 visit of the double-blind trials that would make further GT treatment contraindicated, or for any other condition that would put the patient at unacceptable risk for participation, per investigator judgment.

\subsection{Safety and Efficacy Assessments}

The primary objective of the open-label extension was the assessment of long-term safety. Safety was assessed through treatment-emergent adverse events (TEAEs), local skin reactions (LSRs), laboratory testing, vital signs, and physical examination. Patients were asked about adverse events (AEs) in a non-specific manner using open-ended questions; specific inquiry and evaluation regarding reported AEs were to be conducted when applicable. Local skin reaction evaluation was a static assessment of expected events associated with topically applied products and included burning/stinging, pruritus, edema, erythema, dryness, and scaling; LSRs were graded as mild, moderate, or severe at each clinical visit. Treatment-emergent adverse events were evaluated through week 45 and LSRs through week 44 of the openlabel extension; events reported are those with an onset after the first application of GT in the open-label extension. Discontinuations because of a lack of efficacy were captured under the category of withdrawn consent. Treatment-emergent adverse events of special interest were defined based on association with anticholinergic compounds and potential for serious medical consequences; the Medical Dictionary for Regulatory Activities preferred terms that were pre-specified as TEAEs of special interest were vision blurred, mydriasis, pupils unequal, and hypermetropia and the following terms for symptoms of urinary hesitancy/retention: nocturia, pollakiuria, urinary hesitation, urinary retention, urinary obstruction, and urine flow decreased. Patients with symptoms suggestive of urinary retention were to be evaluated for its clinical course, and for symptoms of urinary obstruction, patients were to be referred to a urologist or for emergency care. Patients who complained of blurry vision were to be carefully queried about the onset of symptoms. If there was no history of inadvertent introduction of study drug into the eye, the patient was to be evaluated to rule out any serious acute condition. If blurry vision continued for $>24 \mathrm{~h}$, the patient was to be evaluated by an ophthalmologist or referred to emergency care. Dose interruptions were allowed if a patient experienced intolerable treatment-related AEs and mandated for treatment-related blurry vision and urinary retention/hesitancy-related symptoms.

Descriptive efficacy assessments in the open-label extension included three outcomes that were among those also used in the double-blind lead-in trials $[9,10]$. Sweat production was measured gravimetrically at day 1 (week 4 visit of the double-blind trials) and week 44/ET over a 5-min period under temperature- and humidity-controlled conditions, and when possible, measurements were conducted at approximately the same time of day for a given patient. Disease severity was assessed by patients using a hyperhidrosisspecific patient-reported outcome measure, the HDSS. This self-reported questionnaire assesses disease severity on a scale of 1 (never noticeable/never interferes with daily activities) to 4 (intolerable/always interferes with daily activities) [13]. The HDSS was assessed on day 1 (week 4 of the double-blind trials) and weeks 2, 4, 8, 12, 16, 20, 28, 36, and 44/ET. Quality of life was assessed using the Dermatology Life Quality Index (DLQI; patients aged $>16$ years) and children's version (CDLQI; patients aged $\leq 16$ years) [14]. Higher DLQI/CDLQI scores (0-30 numeric rating scale) indicate lower quality of life. The DLQI/CDLQI were assessed on day 1 (week 4 of the double-blind trials) and weeks 20 and 44/ET of the open-label extension.

\subsection{Statistical Analysis}

All analyses of the open-label extension data were performed on the safety population (i.e., patients who received one or more confirmed open-label dose of GT and had one or more study assessment) using SAS ${ }^{\circledR}$ version 9.3 or later with no imputation for missing data. No inferential statistics were pre-specified for the safety and efficacy outcomes of the study, and assessments were summarized with descriptive statistics. There was no imputation of missing data for the open-label extension, and pre-specified efficacy analyses were conducted using observed data at week 44/ET (patients who completed 44 weeks of open-label GT and patients who terminated the study early), including median percent change from baseline in gravimetrically measured sweat production, HDSS improvement from baseline by grade (i.e., 1, 2 , and 3 grade) and responder rate (i.e., $\geq 2$-grade improvement from baseline), and mean DLQI/CDLQI score change from baseline. For the HDSS responder rate, a post hoc analysis of data at week 44 was performed for those patients who completed a full 44 weeks of open-label GT treatment. Gravimetric measurement of sweat production can be highly variable even under controlled conditions; therefore, median 
values, which are less influenced by skewed data, were included. Baseline for efficacy assessments in the open-label extension was week 0 of the double-blind trials.

A post hoc analysis was performed to evaluate the longterm treatment response of patients who had received GT during the double-blind lead-in trials and remained on GT into the open-label extension vs. patients newly exposed to GT in the open-label extension because they had received VEH in the double-blind trials. For this analysis, median percent change in gravimetric sweat production, HDSS responder rate, and mean DLQI score change from baseline were analyzed descriptively post hoc according to randomized treatment assignment in the double-blind trials. In addition, the timing of onset of TEAEs and drug withdrawal because of TEAEs were evaluated post hoc according to incidence during weeks $0-4,>4-12,>12-24,>24-36$, and $>36$ to the end of the study and by double-blind treatment assignment.

\section{Results}

\subsection{Patient Disposition, Demographics, and Baseline Disease Characteristics}

Of the 651 patients who completed the double-blind lead-in trials, $564(86.6 \%)$ continued into the open-label extension. Of those 564 patients, 369 (65.4\%) had received GT and 195 (34.6\%) had received VEH during double-blind treatment (Fig. 1). A total of 226 (40.1\%) patients completed week 44 . The most common reasons for discontinuation were early study termination by the sponsor, per protocol, once study objectives were achieved (106/564 [18.8\%]), lost to follow-up (92/564 [16.3\%]), withdrawal of consent (82/564 [14.5\%]), and TEAEs (44/564 [7.8\%]). Only three patients discontinued because of a lack of efficacy (included in the 'withdrawal of consent' category). Of the 564 enrolled patients, 13 had no post-baseline assessment and one did not receive the study drug; therefore, the safety population comprised 550 patients, with $55.3 \%$ female, $83.3 \%$ white, mean age of 33.0 years, and mean body mass index of $27.3 \mathrm{~kg} / \mathrm{m}^{2}$ at the start of the double-blind trials (Table 1). At baseline of the open-label extension (week 4 of the double-blind trials), patients entering the open-label extension who had been taking GT during the double-blind trials $(\mathrm{GT} \rightarrow \mathrm{GT}$ ) had lower sweat production and HDSS and DLQI/CDLQI scores than patients entering the open-label extension who had been treated with VEH for the previous 4 weeks $(\mathrm{VEH} \rightarrow \mathrm{GT}$ ) [Table 1 of the ESM], consistent with the significant improvement in these efficacy measures observed with GT in the double-blind trial population at week $4[9,10]$.

\subsection{Safety}

Over 44 weeks of treatment in the open-label extension, $329 / 550$ (59.8\%) patients reported one or more TEAE (Table 2); most were mild or moderate in severity. The most frequently reported TEAEs were dry mouth (16.9\%), vision blurred (6.7\%), and application-site pain (6.4\%). Of the seven (1.3\%) patients who reported serious TEAEs (one each: infectious colitis, affective disorder, suicide attempt, unilateral mydriasis, chest pain, concussion, diverticulitis),
Fig. 1 Patient disposition. ${ }^{\mathrm{a}} 651$ of 697 patients $(93.4 \%)$ randomized to treatment in ATMOS-1 and ATMOS-2 completed the ATMOS studies. ${ }^{\mathrm{b}}$ Sponsor terminated the study early, per protocol, when the study objective of 100 patients receiving treatment for at least 12 months was achieved. $G T$ glycopyrronium tosylate

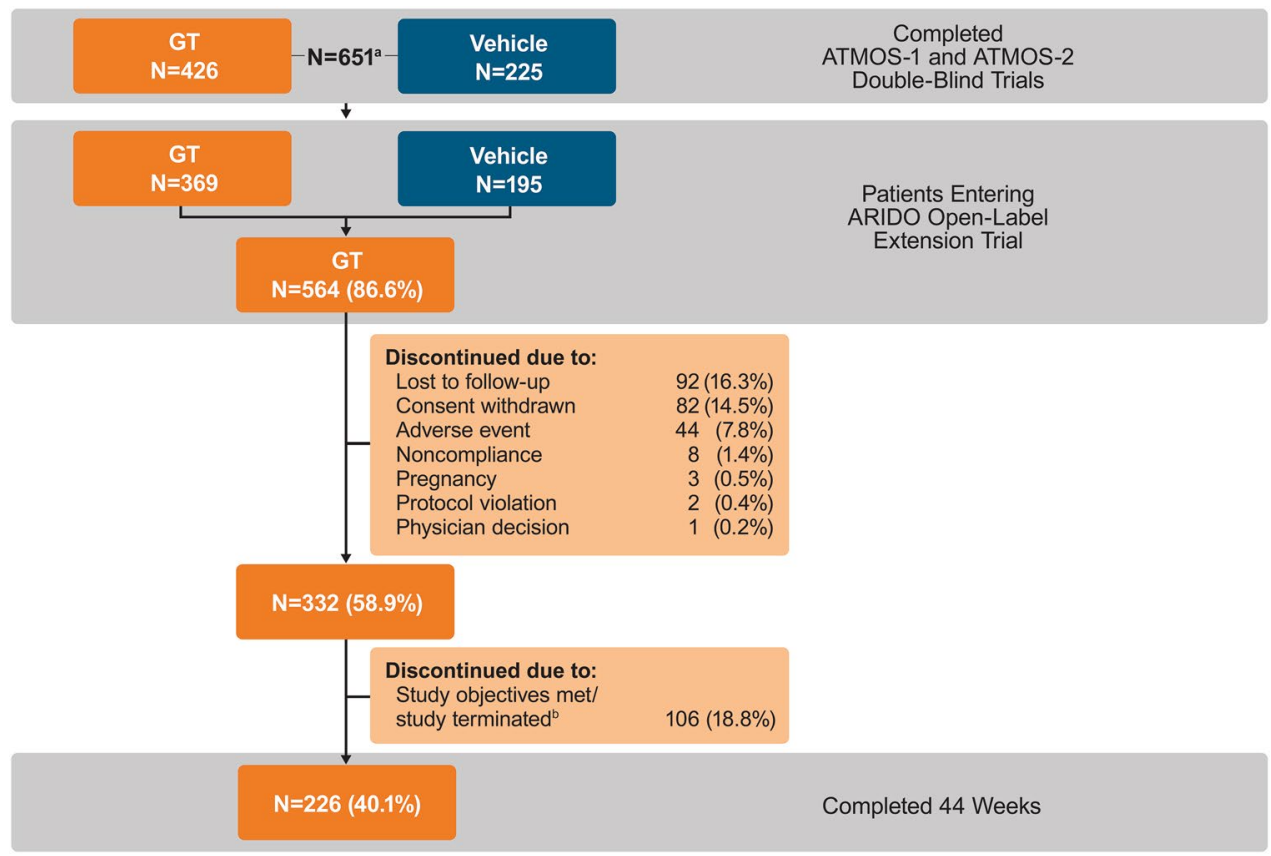


Table 1 Patient demographics and baseline disease characteristics (week 0 of double-blind trials)

\begin{tabular}{ll}
\hline & GT \\
& $N=550$ \\
\hline Demographics & \\
Age (years), mean $\pm \mathrm{SD}$ & $33.0 \pm 11.4$ \\
Age group (years), $n(\%)$ & \\
$\quad<16$ & $28(5.1)$ \\
$\geq 16$ & $522(94.9)$ \\
Female, $n(\%)$ & $304(55.3)$ \\
White, $n(\%)$ & $458(83.3)$ \\
Body mass index $\left(\mathrm{kg} / \mathrm{m}^{2}\right)$, mean $\pm \mathrm{SD}$ & $27.3 \pm 5.0$ \\
Baseline disease characteristics & \\
Sweat production $(\mathrm{mg} / 5$ min) & \\
$\quad$ Mean $\pm \mathrm{SD}^{\mathrm{a}}$ & $164.7 \pm 145.0$ \\
$\quad$ Median & 116.9 \\
HDSS, $n(\%)^{\mathrm{b}, \mathrm{c}}$ & \\
Grade 3 & $348(63.3)$ \\
Grade 4 & $201(36.5)$ \\
DLQI, mean $\pm \mathrm{SD}^{\mathrm{d}}$ & $11.4 \pm 5.9$ \\
CDLQI, mean $\pm \mathrm{SD}^{\mathrm{e}}$ & $8.9 \pm 5.4$ \\
\hline
\end{tabular}

The safety population includes patients receiving $\geq 1$ dose of GT and having $\geq 1$ post-baseline assessment in the open-label extension

$C D L Q I$ children's DLQI, DLQI Dermatology Life Quality Index, GT glycopyrronium tosylate, HDSS Hyperhidrosis Disease Severity Scale, $S D$ standard deviation

${ }^{a}$ Gravimetrically measured average from the left and right axillae

${ }^{\mathrm{b}} \mathrm{HDSS}$ grade $\geq 3$ was an inclusion criterion

${ }^{\mathrm{c}} N=549 ; 1$ patient entered ATMOS-2 with $\operatorname{HDSS}=2$, which was a protocol violation

${ }^{\mathrm{d}}$ Patients aged $>16$ years, $n=512$

${ }^{\text {e}}$ Patients aged $\leq 16$ years, $n=37$

only one was considered by the investigator as related to treatment (mydriasis; dose was not changed but the patient withdrew from the study because of relocation).

Most TEAEs were related to anticholinergic activity and were mild or moderate in severity and infrequently led to study discontinuation. The most frequently reported anticholinergic-related TEAEs were dry mouth (16.9\%), vision blurred $(6.7 \%)$, mydriasis $(5.3 \%)$, urinary hesitation (4.2\%), nasal dryness (3.6\%), and dry eye (2.9\%). A total of 37 patients reported 45 vision blurred events; 40 (88.9\%) were bilateral. A total of 29 patients reported 37 mydriasis events; 31 (83.8\%) were unilateral. Management of TEAEs of special interest (vision blurred, mydriasis, and symptoms of urinary hesitancy/retention) included dose interruption, dosing frequency alteration (e.g., every-other-day dosing), drug withdrawal, and no action. Treatment-emergent adverse events of special interest infrequently led to drug withdrawal, and most events were managed by dose interruption or no action (Fig. 2 of the ESM) and resolved within 3-14 days of
Table 2 Safety overview and treatment-emergent adverse events (TEAEs) in the open-label trial to week 45/end of study

\begin{tabular}{lc}
\hline$n(\%)$ & $\mathrm{GT}$ \\
& $N=550$ \\
\hline Any TEAE & $329(59.8)$ \\
Any serious TEAE & $7(1.3)^{\mathrm{a}}$ \\
Discontinuations because of TEAE & $44(8.0)$ \\
Deaths & 0 \\
TEAEs reported in $\geq 5 \%$ of patients & \\
Dry mouth & $93(16.9)^{\mathrm{b}}$ \\
Vision blurred & $37(6.7)^{\mathrm{b}, \mathrm{c}}$ \\
Application-site pain & $35(6.4)$ \\
Nasopharyngitis & $32(5.8)$ \\
Mydriasis & $29(5.3)^{\mathrm{b}, \mathrm{d}}$ \\
Anticholinergic TEAEs reported in $>2 \%$ of patients & \\
Dry mouth & $93(16.9)^{\mathrm{b}}$ \\
Vision blurred & $37(6.7)^{\mathrm{b}, \mathrm{c}}$ \\
Mydriasis & $29(5.3)^{\mathrm{b}, \mathrm{d}}$ \\
Urinary hesitation & $23(4.2)$ \\
Nasal dryness & $20(3.6)$ \\
Dry eye & $16(2.9)$ \\
\hline & Any TEAE \\
\hline TEAE by intensity & $N=329$ \\
Mild & \\
Moderate & $148(45.0)$ \\
Revere & $153(46.5)$ \\
Not related & $28(8.5)$ \\
Related & $131(39.8)$ \\
\hline & $198(60.2)$ \\
\hline
\end{tabular}

Safety outcomes from the first application of the study drug in the open-label trial are reported. The safety population includes patients receiving $\geq 1$ dose of GT and having $\geq 1$ post-baseline assessment in the open-label extension. Numbers in the table represent the number of patients reporting $\geq 1$ TEAE

$G T$ glycopyrronium tosylate

${ }^{a}$ Infectious colitis, affective disorder, suicide attempt, unilateral mydriasis, chest pain, concussion, diverticulitis; only mydriasis was considered by the investigator as related to treatment (dose was not changed but patient withdrew from study because of moving domiciles)

${ }^{\mathrm{b}}$ Dry mouth, vision blurred, and mydriasis appear twice in the table as they meet criteria for common TEAEs and are also associated with anticholinergic use

${ }^{\mathrm{c}}$ Thirty-seven patients reported a total of 45 vision blurred events; 40 (88.9\%) were bilateral

${ }^{\mathrm{d}}$ Twenty-nine patients reported a total of 37 mydriasis events; 31 $(83.8 \%)$ were unilateral

onset. Of the 17 patients who discontinued the study because of a TEAE of special interest, most (76.5\% [13/17]) did not have a dose interruption or dosing frequency alteration prior to discontinuation. Only one TEAE of special interest was 
Table 3 Treatment-emergent adverse events (TEAEs) by time of onset in the open-label trial to week 45/end of study (EOS)

\begin{tabular}{|c|c|c|c|c|c|}
\hline$N(\%)$ & $\begin{array}{l}0-4 \text { weeks } \\
N=550\end{array}$ & $\begin{array}{l}>4 \text { to } 12 \text { weeks } \\
N=537\end{array}$ & $\begin{array}{l}>12 \text { to } 24 \text { weeks } \\
N=479\end{array}$ & $\begin{array}{l}>24 \text { to } 36 \text { weeks } \\
N=417\end{array}$ & $\begin{array}{l}>36 \text { weeks to EOS } \\
N=365\end{array}$ \\
\hline Any TEAE & $176(32.0)$ & $148(27.6)$ & $102(21.3)$ & $78(18.7)$ & $59(16.2)$ \\
\hline $\begin{array}{l}\text { Drug withdrawal because } \\
\text { of TEAE }\end{array}$ & $21(3.8)$ & $14(2.6)$ & $12(2.5)$ & $3(0.7)$ & $1(0.3)$ \\
\hline \multicolumn{6}{|c|}{ TEAEs reported in $\geq 5 \%$ of patients } \\
\hline Dry mouth ${ }^{\mathrm{a}}$ & $59(10.7)$ & $23(4.3)$ & $19(4.0)$ & $15(3.6)$ & $5(1.4)$ \\
\hline Vision blurred $^{\mathrm{a}, \mathrm{b}}$ & $11(2.0)$ & $14(2.6)$ & $7(1.5)$ & $5(1.2)$ & $4(1.1)$ \\
\hline Application-site pain & $16(2.9)$ & $9(1.7)$ & $5(1.0)$ & $6(1.4)$ & $3(0.8)$ \\
\hline Nasopharyngitis & $14(2.5)$ & $9(1.7)$ & $4(0.8)$ & $5(1.2)$ & $3(0.8)$ \\
\hline Mydriasis $^{\mathrm{a}, \mathrm{c}}$ & $8(1.5)$ & $8(1.5)$ & $9(1.9)$ & $5(1.2)$ & $2(0.5)$ \\
\hline \multicolumn{6}{|c|}{ Anticholinergic TEAEs reported in $>2 \%$ of patients } \\
\hline Dry mouth ${ }^{\mathrm{a}}$ & $59(10.7)$ & $23(4.3)$ & $19(4.0)$ & $15(3.6)$ & $5(1.4)$ \\
\hline Vision blurred $^{\mathrm{a}, \mathrm{b}}$ & $11(2.0)$ & $14(2.6)$ & $7(1.5)$ & $5(1.2)$ & $4(1.1)$ \\
\hline Mydriasis $^{\mathrm{a}, \mathrm{c}}$ & $8(1.5)$ & $8(1.5)$ & $9(1.9)$ & $5(1.2)$ & $2(0.5)$ \\
\hline Urinary hesitation & $14(2.5)$ & $4(0.7)$ & $4(0.8)$ & $2(0.5)$ & $1(0.3)$ \\
\hline Nasal dryness & $13(2.4)$ & $2(0.4)$ & $3(0.6)$ & $5(1.2)$ & $1(0.3)$ \\
\hline Dry eye & $10(1.8)$ & $1(0.2)$ & $3(0.6)$ & $3(0.7)$ & $2(0.5)$ \\
\hline
\end{tabular}

Safety outcomes from the first application of the study drug in the open-label trial are reported. The safety population includes patients receiving $\geq 1$ dose of GT and having $\geq 1$ post-baseline assessment in the open-label extension. Numbers in the table represent the number of patients reporting $\geq 1$ TEAE that started within the indicated time period

GT glycopyrronium tosylate

${ }^{a}$ Dry mouth, vision blurred, and mydriasis appear twice in the table as they meet criteria for common TEAEs and are also associated with anticholinergic use

${ }^{\mathrm{b}}$ Thirty-seven patients reported a total of 45 vision blurred events; 40 (88.9\%) were bilateral

${ }^{\mathrm{c}}$ Twenty-nine patients reported a total of 37 mydriasis events; 31 (83.8\%) were unilateral

severe (vision blurred), and the event resolved upon drug interruption. After treatment re-initiation, the patient experienced three TEAEs of moderate severity (dry mouth, pharyngitis, and bilateral vision blurred), all deemed related to the study drug, and all three resolved upon discontinuation of the study drug.

The onset of both common and anticholinergic TEAEs occurred mainly in the first 12 weeks of treatment in the open-label extension and decreased thereafter (Table 3), indicating that TEAEs did not increase with a longer duration of exposure. Similarly, rates of drug withdrawal because of TEAEs remained relatively stable over the study course (Table 3). The incidence of dry mouth was higher in the first 4 weeks of the open-label extension for patients who were newly exposed to GT in the open-label extension (i.e., had received VEH during the double-blind lead-in trials), though rates of drug withdrawal because of TEAEs were not higher in this patient population (Table 2 of the ESM). Most patients ( $n=371[67.5 \%]$ ) had no LSRs; of those patients that reported LSRs, erythema $(n=116$ [21.1\%]), burning/ stinging ( $n=73[13.3 \%])$, and pruritus $(n=68[12.4 \%])$ were most common. Local skin reactions were predominantly mild or moderate in intensity (Fig. 2), the frequency and severity did not increase over the course of the open-label extension, and a total of 11 patients had LSRs that were associated with study discontinuation. No clinically meaningful changes in laboratory testing, vital signs, and physical examination were observed over 44 weeks of treatment in the open-label extension.

\subsection{Descriptive Efficacy Measures}

Through week 44/ET of the open-label extension (up to 48 weeks of GT from the start of the double-blind leadin trials), GT-treated patients maintained improvements in efficacy measures, including sweat production, HDSS responder rate, and DLQI/CDLQI. From baseline of the double-blind trials to week 44/ET in the open-label extension, sweat production decreased by $71.3 \%$, which was maintained from a similar decrease of $74.3 \%$ in GT-treated patients after 4 weeks of treatment in the double-blind trials (Fig. 3a). At week 44/ET in the open-label extension, HDSS improved from baseline of the double-blind trials by 1,2, and 3 grades in $30.9 \%, 46.7 \%$, and $16.5 \%$ of patients, respectively, which was maintained from a similar improvement at week 4 of the double-blind trials (Fig. 4a). The proportion of HDSS responders (i.e., $\geq 2$-grade improvement) was consistent over the study course (Fig. 4b) and similar to that 


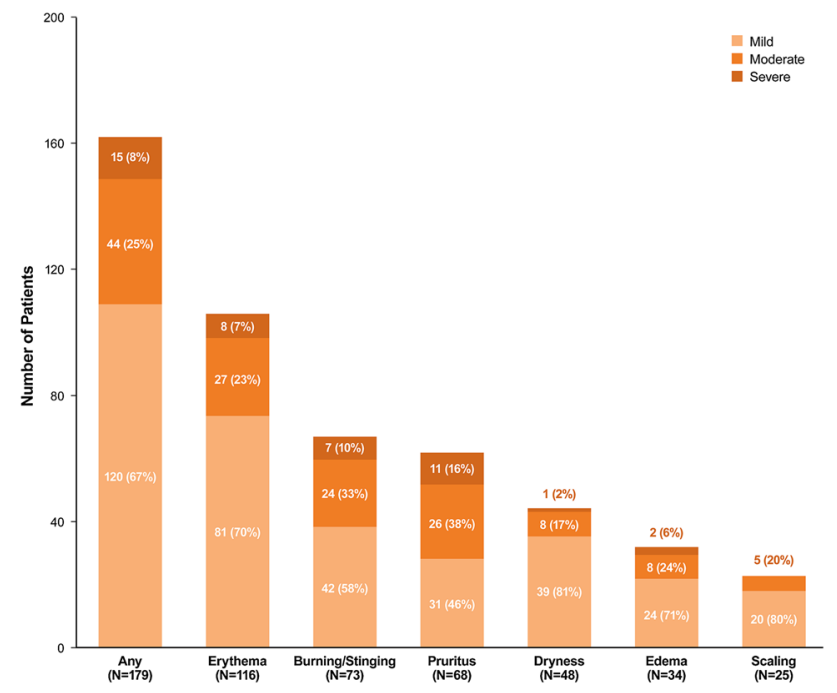

Fig. 2 Local skin reactions (LSRs) in the open-label trial to week 44/ end of treatment/early termination (ET) $[n=550]$. The safety population includes patients receiving one or more doses of glycopyrronium tosylate (GT) and having one or more post-baseline assessments in the open-label extension. Patients were counted as having an LSR if any post-baseline assessment was mild, moderate, or severe; percentages represent the proportion of patients who had LSR events with a maximum post-baseline severity of mild, moderate, or severe

observed after 4 weeks of GT treatment in the double-blind trials (59.1\%) [9]. The HDSS responder rate determined post hoc for patients who completed a full 44 weeks of openlabel GT treatment $(65.1 \%)$ was similar to the responder rate seen in the pre-specified week 44/ET analysis (63.2\%). As measured by the DLQI and CDLQI, improvements in quality of life from baseline of the double-blind trials to week 44/ET of the open-label extension (reflected by mean score decreases of $8.7 \pm 6.2$ and $6.2 \pm 4.9$, respectively) were maintained from mean decreases of $8.4 \pm 6.0$ and $8.1 \pm 5.4$, respectively, observed in GT-treated patients after 4 weeks of treatment in the double-blind trials (Fig. 5a, b).

Long-term treatment response in patients remaining on GT from the double-blind trials into the open-label extension (GT $\rightarrow$ GT) and in those patients newly exposed to GT in the open-label extension (VEH $\rightarrow \mathrm{GT}$ ) was evaluated. At week 44 of the open-label extension, patients who had been taking VEH in the double-blind trials showed a substantial reduction in sweat production compared with week 4 of the double-blind trials (Fig. 3b). By week 2 of the open-label extension, the HDSS response rate was $62.8 \%$ (223/355) for GT $\rightarrow$ GT (patients who had been taking GT in the doubleblind trials) vs. 55.7\% (102/183) for VEH $\rightarrow$ GT, representing a marked increase in response for those patients who had been taking VEH in the double-blind trials. The HDSS response was maintained and slightly increased over the course of the open-label extension for both groups, with $67.7 \%(126 / 186)$ for GT $\rightarrow$ GT and $60.4 \%$ (64/106) for
$\mathrm{VEH} \rightarrow \mathrm{GT}$ at week 44/ET (Fig. 4c). Analyses of the DLQI mean score change showed a similar trend (Fig. 5c).

\section{Discussion}

Axillary hyperhidrosis is a chronic condition that requires long-term treatment as symptoms often present in adolescence and persist throughout a patient's lifetime [15]. Patient experience with adverse events (both onset and time course) and the long-term effectiveness are therefore important considerations when managing treatment choices.

The long-term safety and efficacy findings presented here from the 44-week, open-label extension study of GT in primary axillary hyperhidrosis are consistent with the individual and pooled data from the two replicate, doubleblind, placebo-controlled, phase III, lead-in studies [9]. Once-daily application of GT was well tolerated for up to 48 weeks (4 weeks in the double-blind trials and 44 weeks in the open-label extension) with TEAEs that were mostly related to anticholinergic activity and largely mild or moderate in severity. Importantly, most TEAEs, including anticholinergic TEAEs, occurred within the first 12 weeks of treatment in the open-label extension, and decreased thereafter, indicating that the incidence of TEAEs decreased over time, particularly for dry mouth. Consistent with this observation in the overall population, patients newly exposed to GT in the open-label extension (who had received VEH during the double-blind trials) had a much higher incidence of dry mouth in the first 4 weeks of the open-label extension compared with those continuing on GT from double-blind into open-label treatment. A reduction in TEAEs reported over time may be due to several factors, including patient selection as those experiencing TEAEs discontinued, increased time length between visits, increased patient experience with drug application, and/or acclimation to adverse events. Few (8.0\%) patients discontinued because of a TEAE, and the rate of discontinuation because of TEAEs was relatively stable as the study progressed. Most TEAEs of special interest (mydriasis, vision blurred, symptoms of urinary hesitancy/ retention) were transient and managed by GT interruption or no action.

While approximately one third of patients reported LSRs, most were mild or moderate in intensity, did not increase in frequency and severity over the course of the open-label extension, and rarely resulted in study discontinuation. Overall, safety results were consistent with the safety profile observed in prior GT studies [9]. No new or unexpected findings were noted, highlighting the long-term treatment potential of GT for primary axillary hyperhidrosis. Topical administration of anticholinergics is expected to reduce overall drug exposure and mitigate adverse event risk compared with oral agents [16]. Proper application and adequate hand 

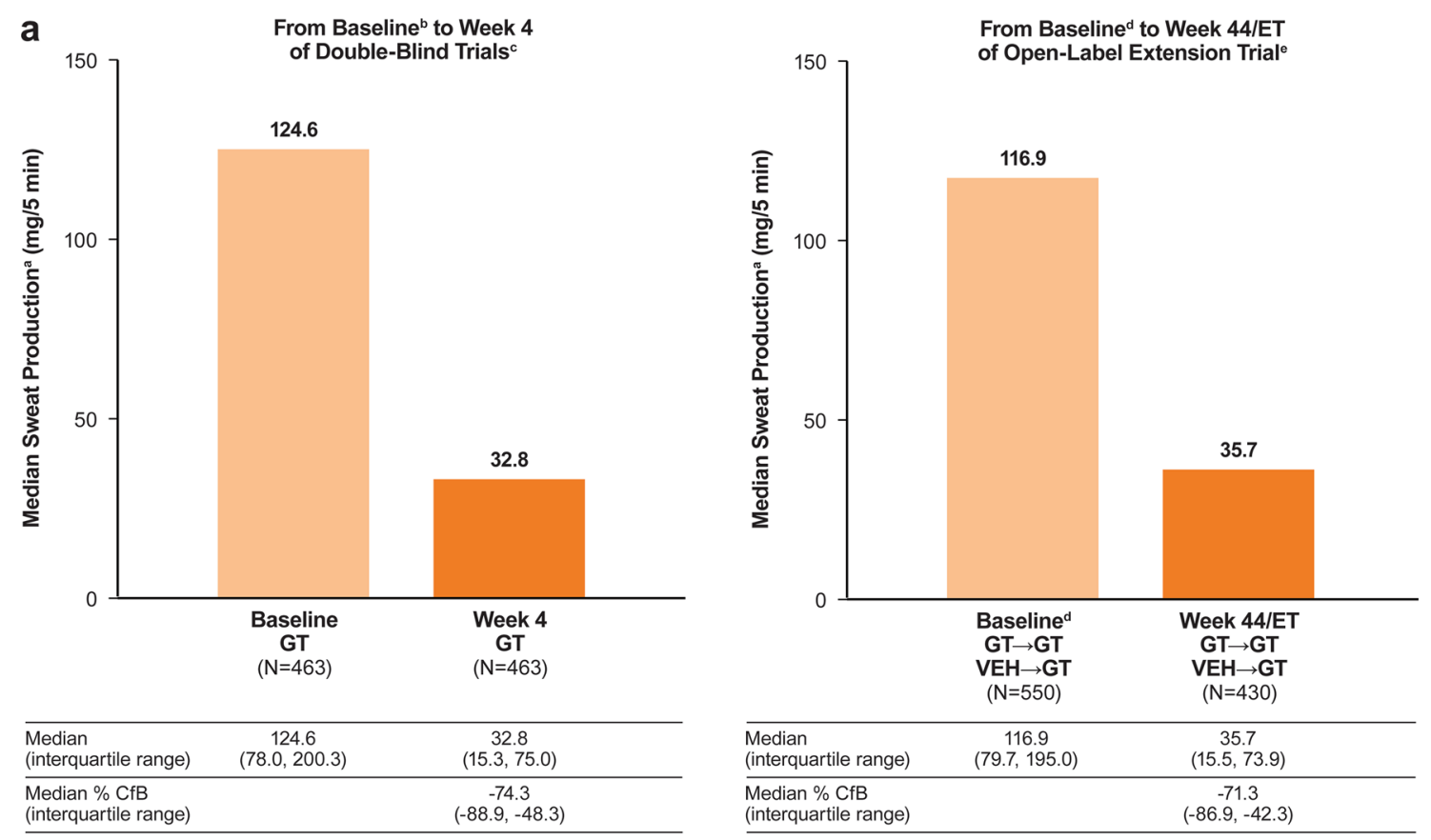

b

From Baseline ${ }^{d}$ to Week 4 of Double-Blind Trials and Week 44/ET of Open-Label Extension Trial ${ }^{e}$

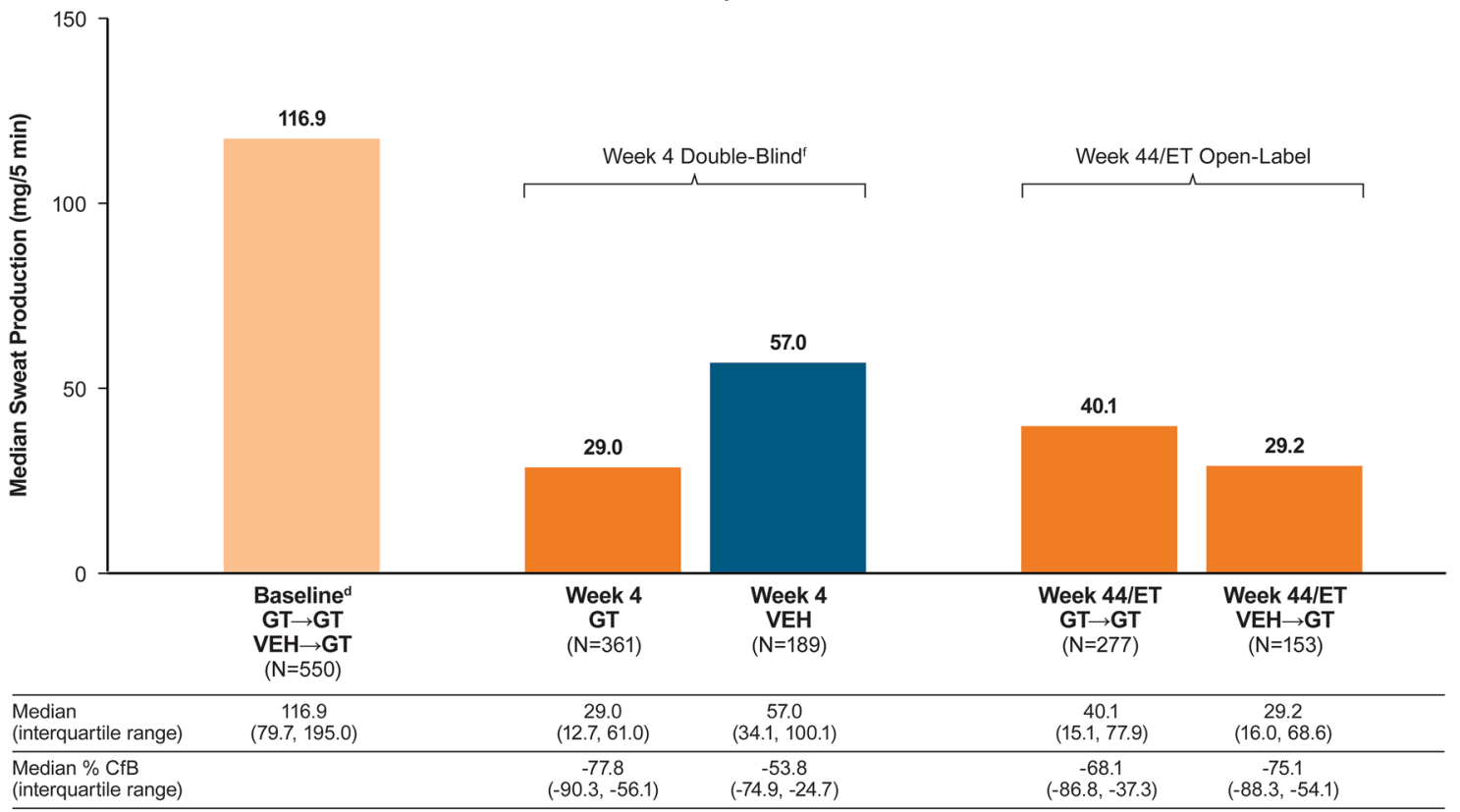

Fig. 3 Sweat production: change from baseline (week 0 of doubleblind trials) for (a) all glycopyrronium tosylate (GT)-treated patients and (b) analysis by double-blind treatment assignment. ${ }^{\text {a }}$ Sweat production values reported were gravimetrically measured averages from the left and right axillae. ${ }^{\mathrm{b}}$ Baseline in the double-blind ATMOS-1/ ATMOS-2 trials for GT-treated patients only. ${ }^{\text {cPooled ATMOS-1/ }}$ ATMOS-2 intent-to-treat (ITT) population (all randomized patients dispensed the study drug in ATMOS-1/ATMOS-2); missing data were imputed by Markov chain Monte Carlo. ${ }^{\mathrm{d}}$ Baseline in the doubleblind ATMOS-1/ATMOS-2 trials for vehicle (VEH)- and GT-treated patients who continued into the open-label extension and received GT treatment thereafter. ${ }^{\mathrm{e}}$ Open-label safety population (patients receiv- ing one or more doses of GT and having one or more post-baseline assessments in the open-label extension); the pre-specified analysis of week 44/end of treatment/early termination (ET) included patients who completed 44 weeks of open-label GT and patients who terminated the study early; there was no imputation for missing data. ${ }^{\mathrm{f}} \mathrm{Data}$ are for week 4 of the double-blind ATMOS-1/ATMOS-2 trials for those patients entering the open-label extension. $C f B$ change from baseline, $G T \rightarrow G T$ patients assigned to GT in the double-blind trials who continued taking GT in the open-label extension, $V E H \rightarrow G T$ patients assigned to VEH in the double-blind trials who were newly exposed to GT in the open-label extension 
a

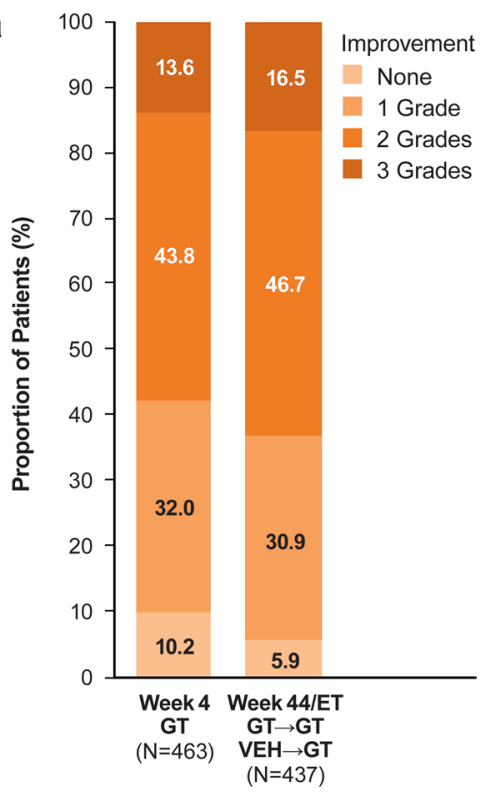

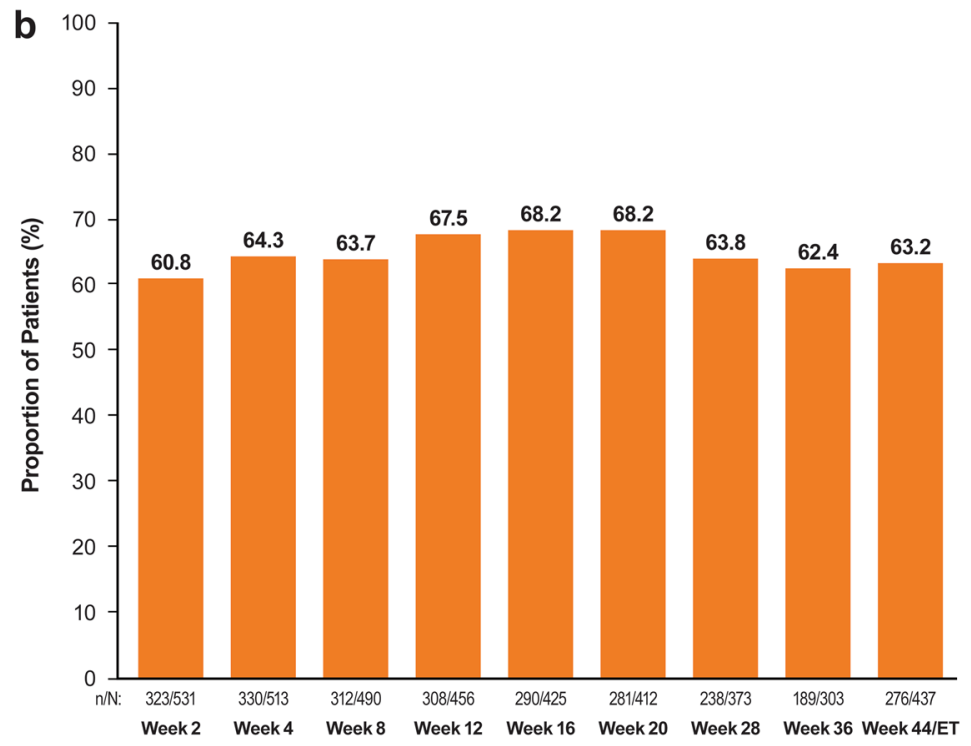

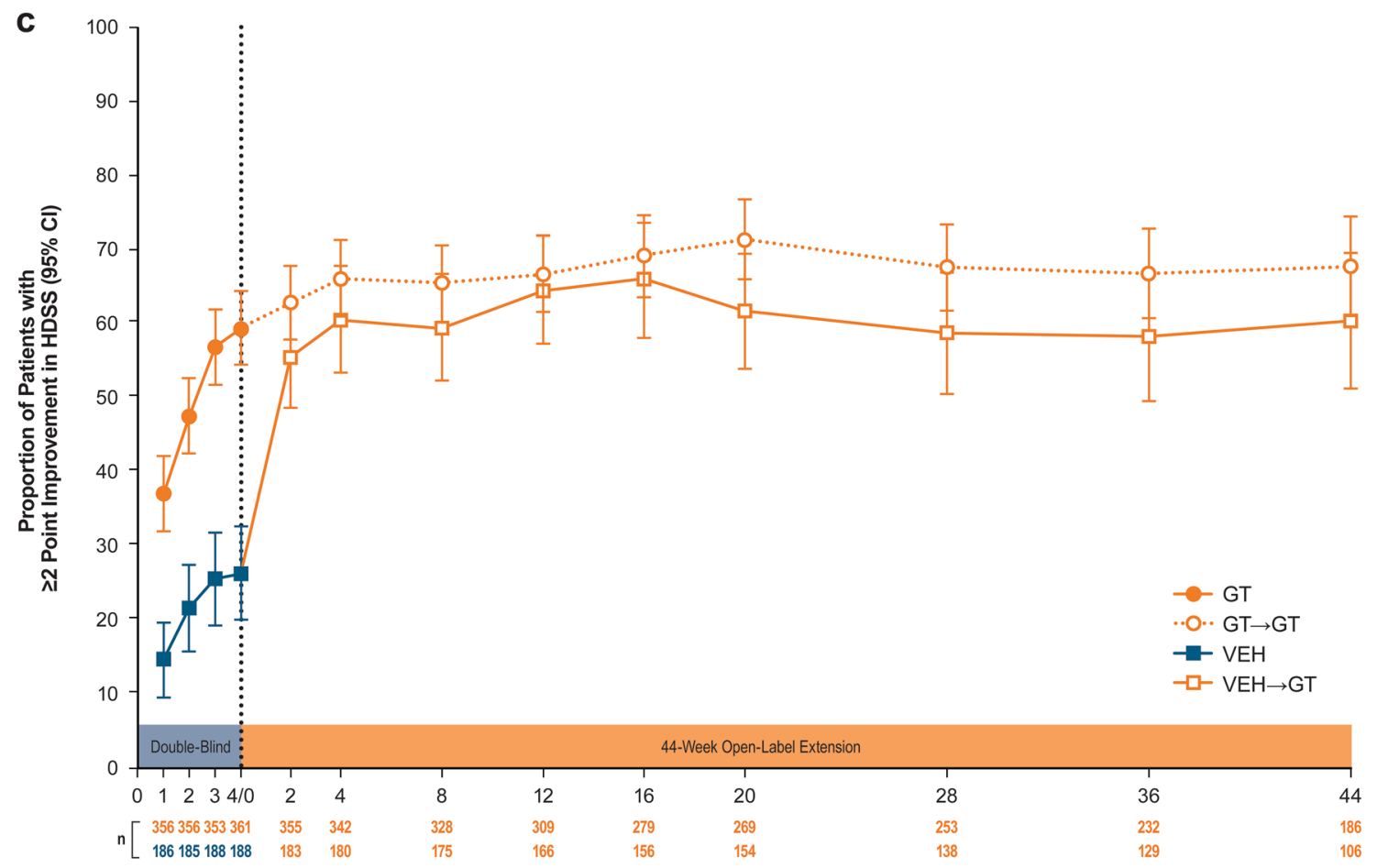

Week

Fig. 4 Hyperhidrosis Disease Severity Scale (HDSS) improvement from baseline (week 0 of double-blind trials) for (a) proportion of glycopyrronium tosylate (GT)-treated patients with $0-, 1-, 2-$, or 3-grade HDSS improvement from baseline to week 4 of doubleblind trials and week 44/end of treatment/early termination (ET) of the open-label extension trial, (b) HDSS responder rate $(\geq 2$-grade improvement from baseline) in the open-label trial to week 44/ ET, and (c) analysis by double-blind treatment assignment: HDSS responder rate to week 44 . The open-label safety population includes patients receiving one or more doses of GT and having one or more post-baseline assessments in the open-label extension. The prespecified analysis of week 44/ET included patients who completed 44 weeks of open-label GT and patients who terminated the study early; there was no imputation for missing data. $G T \rightarrow G T$ patients assigned to GT in the double-blind trials who continued on GT in the open-label extension, $V E H$ vehicle, $V E H \rightarrow G T$ patients assigned to VEH in the double-blind trials who were newly exposed to GT in the open-label extension 

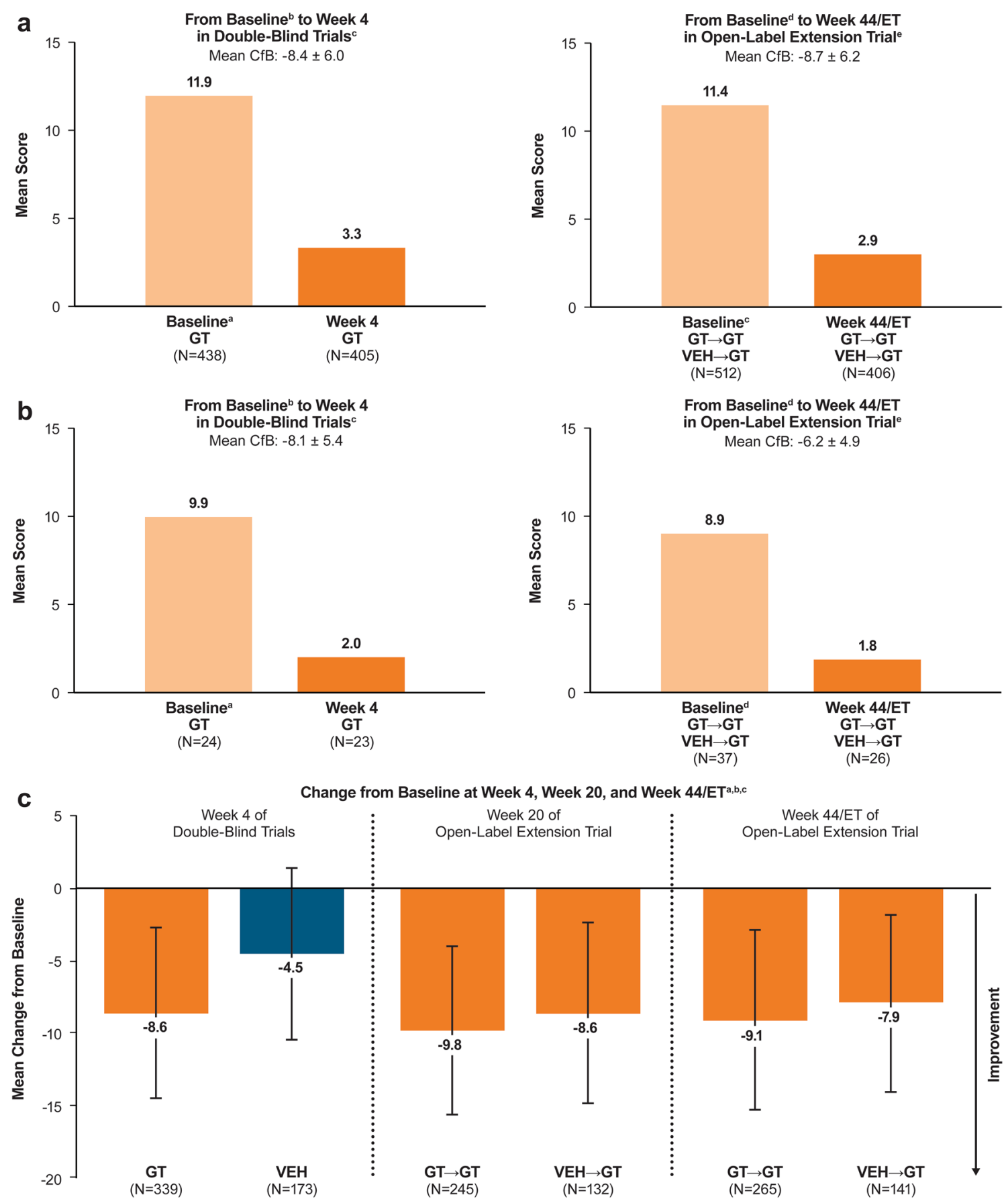

Fig. 5 Dermatology Life Quality Index (DLQI) and children's DLQI (CDLQI) mean scores for (a) change from baseline (week 0 of double-blind trials) in DLQI ${ }^{\mathrm{a}}$, (b) change from baseline (week 0 of double-blind trials) in $\mathrm{CDLQI}^{\mathrm{f}}$, and (c) analysis by double-blind

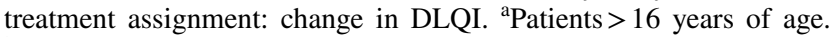
${ }^{\mathrm{b}}$ Baseline in ATMOS-1/ATMOS-2 for glycopyrronium tosylate (GT)treated patients only. ${ }^{\mathrm{c}} \mathrm{ATMOS}-1 / \mathrm{ATMOS}-2$ intent-to-treat population (all randomized patients dispensed the study drug in ATMOS-1/ ATMOS-2). ${ }^{\mathrm{d}}$ Baseline in ATMOS-1/ATMOS-2 for vehicle (VEH)and GT-treated patients who continued into ARIDO and received GT treatment thereafter. ${ }^{\text {e}}$ Open-label safety population (patients receiv- ing one or more doses of GT and having one or more post-baseline assessments in the open-label extension); the pre-specified analysis of week 44/end of treatment/early termination (ET) included patients who completed 44 weeks of open-label GT and patients who terminated the study early; there was no imputation of missing data. ${ }^{\mathrm{f}} \mathrm{Pa}-$ tients $\leq 16$ years of age. $C f B$ change from baseline, $G T \rightarrow G T$ patients assigned to GT in the double-blind trials who continued on GT in the open-label extension, $V E H \rightarrow G T$ patients assigned to VEH in the double-blind trials who were newly exposed to GT in the open-label extension 
washing is required following GT application to minimize the risk of inadvertent transfer of the drug from the hands to other areas of the body, including the eyes.

In the 4-week, double-blind, lead-in trials, GT treatment resulted in significant improvements in sweating severity, sweat production, and quality of life, which were evident as early as week 1 [9]. Importantly, these improvements were maintained during long-term treatment with GT. At the end of treatment in the open-label extension, patients continued to have reduced gravimetrically measured sweat production, reduced disease severity as measured by the HDSS, and improved quality of life as measured by the DLQI/CDLQI compared with baseline of the double-blind trials. Patients who were newly exposed to GT in the open-label extension showed a response to GT treatment that occurred early, which was similar to results observed for the GT arm of the double-blind, lead-in trials and consistent with the openlabel results observed for the patients originally assigned GT in double-blind trials. Efficacy was maintained or increased throughout the long-term, open-label extension irrespective of randomized treatment in the double-blind trials. Collectively, these data indicate a long-term treatment advantage of GT vs. VEH across a spectrum of outcomes.

A number of study limitations should be recognized when evaluating the data from this open-label extension. As with all open-label extension trials, the interpretation of efficacy and safety outcomes is limited by the lack of a placebo control. In addition, lack of imputation for missing data may lead to an underestimation of the $\mathrm{AE}$ rate and/or an overestimation of efficacy because patients with AEs or who experienced a lack of efficacy may have dropped out during the doubleblind trial phase or early in the open-label extension. The pediatric sample size in this trial was small, and further study in younger patients will be useful. It should be noted that post hoc analyses of the pediatric patient population from the double-blind, lead-in trials [11] and this open-label extension [17] show an advantage with GT across multiple efficacy measures and favorable tolerability, with similar findings in children and adults. Because of the different study designs, it is not advisable to directly compare the present long-term results with those of other drugs used to treat axillary hyperhidrosis, including other anticholinergic medications, and GT efficacy and safety data beyond 48 weeks of treatment cannot be extrapolated from the results of this trial. Given the lifelong chronicity of hyperhidrosis, long-term patient follow-up will be appropriate.

\section{Conclusion}

Glycopyrronium tosylate was generally well tolerated in this open-label extension study, and improvements in efficacy measures were maintained in patients with primary axillary hyperhidrosis when applied once daily to both axillae over a maximum of 48 weeks (double-blind plus open-label). The approval by the US Food and Drug Administration of the topical anticholinergic GT for the treatment of primary axillary hyperhidrosis in patients aged $\geq 9$ years $\left(\mathrm{Qbrexza}^{\mathrm{TM}}\right.$ [glycopyrronium] cloth, $2.4 \%$, for topical use) provides a new, non-invasive, once-daily treatment option for a condition associated with a substantial patient burden, and GT represents a treatment option that can be used effectively over the long term. Glycopyrronium tosylate is considered a first-line treatment option for primary axillary hyperhidrosis [8], and future studies evaluating the effectiveness of GT in real-life settings (e.g., add-on therapy with other hyperhidrosis treatments) and its use for other types of hyperhidrosis (e.g., palmar hyperhidrosis) may further define its optimal clinical use.

Acknowledgements Medical writing support for this manuscript was provided by Ashley A. Skorusa, $\mathrm{PhD}$ and Merrilee Johnstone, $\mathrm{PhD}$ of Prescott Medical Communications Group (Chicago, IL, USA), with financial support from Dermira, Inc.

\section{Compliance with Ethical Standards}

Funding This study was sponsored and funded by Dermira, Inc.

Conflict of interest Dee Anna Glaser is an investigator for Allergan, Galderma, ATACAMA, Brickell Biotech, Inc., Dermira, Inc., Evolus, and Sienna Biopharmaceuticals, Inc. and a consultant for Dermira, Inc. William P. Werschler is a consultant and investigator for Dermira, Inc. Adelaide A. Herbert is a consultant for Dermira, Inc. and an employee of the UTHealth McGovern Medical School, which received compensation from Dermira, Inc. for study participation. Alexander Nast is an employee of Charité - Universitätsmedizin Berlin, which received compensation from Dermira, Inc. for study participation; he was an advisory board member for Boehringer Ingelheim, carried out educational activities for Bayer and Novartis, and received research grants from Eli Lilly and Company, Pfizer, GSK, Plc., and MEDA. Lawrence Green is an investigator for Brickell Biotech, Inc. and an advisory board member and investigator for Dermira, Inc. Richard D. Mamelok is a consultant for Dermira, Inc. John Quiring is an employee of QST Consultations. Janice Drew is an employee of Dermira, Inc. David M. Pariser is a consultant and investigator for Brickell Biotech, Inc., Celgene Corporation, Dermira, Inc., LEO Pharma US, Novartis Pharmaceuticals, Promius Pharmaceuticals, Regeneron, and Valeant Pharmaceuticals International, a consultant for ATACAMA, Biofrontera AG, DUSA Pharmaceuticals, Inc., Sonofi, TDM SurgiTech, Inc., and TheraVida, and an investigator for Abbott Laboratories, Amgen, Asana Biosciences, Dermavant Sciences, Eli Lilly and Company, Merck \& Co., Inc., Novo Nordisk A/S, Ortho Dermatologics, Peplin Inc., Pfizer Inc., Photocure ASA, and Stiefel (as GSK company).

Ethics approval and consent to participate The ARIDO open-label extension study was conducted in the USA and Germany. The trial protocol and informed consent forms were approved by local institutional review boards or independent ethics committees on 10 August, 2015, and the first patient was enrolled on 27 August, 2015. The trial was registered on ClinicalTrials.gov on 18 September, 2015 (NCT02553798) and was carried out in accordance with Good Clinical Practice and the Declaration of Helsinki. 
Open Access This article is distributed under the terms of the Creative Commons Attribution-NonCommercial 4.0 International License (http://creativecommons.org/licenses/by-nc/4.0/), which permits any noncommercial use, distribution, and reproduction in any medium, provided you give appropriate credit to the original author(s) and the source, provide a link to the Creative Commons license, and indicate if changes were made.

\section{References}

1. Doolittle J, Walker P, Mills T, Thurston J. Hyperhidrosis: an update on prevalence and severity in the United States. Arch Dermatol Res. 2016;308:743-9.

2. Grabell DA, Hebert AA. Current and emerging medical therapies for primary hyperhidrosis. Dermatol Ther (Heidelb). 2017;7:25-36.

3. Naumann M, Hamm H, Spalding JR, Kowalski J, Lee J. Comparing the quality of life effects of primary focal hyperhidrosis to other dermatological conditions as assessed by the Dermatology Life Quality Index (DLQI). Value Health. 2003;6:242.

4. Strutton DR, Kowalski JW, Glaser DA, Stang PE. US prevalence of hyperhidrosis and impact on individuals with axillary hyperhidrosis: results from a national survey. J Am Acad Dermatol. 2004;51:241-8.

5. Alvarez MA, Ruano J, Gomez FJ, Casas E, Baamonde C, Salvatierra A, et al. Differences between objective efficacy and perceived efficacy in patients with palmar hyperhidrosis treated with either botulinum toxin or endoscopic thoracic sympathectomy. $\mathbf{J}$ Eur Acad Dermatol Venereol. 2013;27:e282-8.

6. Glaser DA, Hebert A, Pieretti L, Pariser D. Understanding patient experience with hyperhidrosis: a national survey of 1,985 patients. J Drugs Dermatol. 2018;17:392-6.

7. Qbrexza (glycopyrronium) cloth, $2.4 \%$, for topical use [prescribing information]. Menlo Park (CA): Dermira, Inc.; 2018.

8. International Hyperhidrosis Society. Hyperhidrosis treatment algorithms. 2018. Available from: https://www.sweathelp.org/treat ments-hcp/clinical-guidelines/hyperhidrosis-treatment-algorithms .html. Accessed 8 Nov 2018.
9. Glaser DA, Hebert AA, Nast A, Werschler WP, Green L, Mamelok $\mathrm{R}$, et al. Topical glycopyrronium tosylate for the treatment of primary axillary hyperhidrosis: results from the ATMOS-1 and ATMOS-2 phase 3 randomized controlled trials. J Am Acad Dermatol. 2019;80(1):128-38.e2.

10. Pariser DM, Hebert AA, Drew J, Quiring J, Gopalan R, Glaser DA. Topical glycopyrronium tosylate for the treatment of primary axillary hyperhidrosis: patient reported outcomes from the ATMOS-1 and ATMOS-2 phase 3 randomized controlled trials. Am J Clin Dermatol. 2019;20(1):135-45.

11. Hebert AA, Glaser D, Green L, Werschler WP, Forsha DW, Drew $\mathrm{J}$, et al. Glycopyrronium tosylate in pediatric primary axillary hyperhidrosis: post hoc analysis of efficacy and safety findings by age from two phase 3 randomized controlled trials. Pediatr Dermatol. 2019;36(1):89-99.

12. Glaser DA, Hebert AA, Fehnel S, DiBenedetti D, Nelson L, Drew $\mathrm{J}$, et al. Confirmatory psychometric evaluation of the Axillary Sweating Daily Diary: a validated patient-reported outcome measure to assess axillary hyperhidrosis sweating severity [abstract]. J Clin Aesthet Dermatol. 2018;11(5 Suppl.):S16-7.

13. Solish N, Bertucci V, Dansereau A, Hong HC, Lynde C, Lupin M, et al. A comprehensive approach to the recognition, diagnosis, and severity-based treatment of focal hyperhidrosis: recommendations of the Canadian Hyperhidrosis Advisory Committee. Dermatol Surg. 2007;33:908-23.

14. Finlay AY, Khan GK. Dermatology Life Quality Index (DLQI): a simple practical measure for routine clinical use. Clin Exp Dermatol. 1994;19:210-6.

15. Sammons JE, Khachemoune A. Axillary hyperhidrosis: a focused review. J Dermatol Treat. 2017;28:582-90.

16. Staskin DR. Transdermal systems for overactive bladder: principles and practice. Rev Urol. 2003;5(Suppl. 8):S26-30.

17. Hebert AA, Glaser DA, Green L, Werschler WP, Forsha DW, Drew J, et al., editors. Short- and long-term efficacy and safety of glycopyrronium tosylate for the treatment of primary axillary hyperhidrosis: post hoc pediatric subgroup analyses from the phase 3 studies. In: Oral presentation at the 27th international congress of the European academy of dermatology and venereology; 12-16 September, 2018; Paris.

\section{Affiliations}

\section{Dee Anna Glaser ${ }^{1}$ - Adelaide A. Hebert ${ }^{2}$. Alexander Nast ${ }^{3,4}$ - William P. Werschler ${ }^{5}$. Lawrence Green ${ }^{6}$. Richard D. Mamelok ${ }^{7} \cdot$ John Quiring ${ }^{8} \cdot$ Janice Drew $^{9} \cdot$ David M. Pariser $^{10}$}

1 Saint Louis University, 1755 S. Grand Blvd, St. Louis, MO 63104, USA

2 UTHealth McGovern Medical School, Houston, TX, USA

3 Charité-Universitätsmedizin Berlin, Corporate Member of Freie Universität Berlin, Humboldt-Universität zu Berlin, Berlin, Germany

4 Division of Evidence-Based Medicine, Department of Dermatology, Venerology und Allergy, Berlin Institute of Health, Berlin, Germany

5 Premier Clinical Research, Spokane, WA, USA
6 George Washington University School of Medicine, Washington, DC, USA

7 Mamelok Consulting, Palo Alto, CA, USA

8 QST Consultations, Allendale, MI, USA

9 Dermira, Inc., Menlo Park, CA, USA

10 Eastern Virginia Medical School, Virginia Clinical Research, Inc., Norfolk, VA, USA 